striekonzerne mit der Umstellung auf ISO befassen. In diesen beiden Ländern entstehen ebenfalls Q-System-Zertifizierungsstellen.

Es darf somit erfreut festgestellt werden, dass auch in weiteren Teilen der Welt immer grössere Anstrengungen hinsichtlich Qualitätssicherung und der Einführung von integralen Q-Systemen sowie deren Zertifizierung unternommen werden mit dem erklärten Ziel, die Qualitätsfähigkeit der Firmen entscheidend anzuheben.

Alle diese Anstrengungen bezüglich höherer Qualitätsfähigkeit und Zuverlässigkeit, die europa- und weltweit unternommen werden, wirken sich aber teilweise sehr direkt und zum Teil auch indirekt auf die einzelnen Firmen der europäischen Industrie und Wirtschaft aus; dies schon wegen der engen europa- und weltweiten Verknüpfung der Wirtschaft.

Es gilt für die einzelnen Firmen die Herausforderung anzunehmen und die Qualitäts-und Wettbewerbsfähigkeit ganz allgemein zu steigern. Für das einzelne Unternehmen bedeutet dies, die notwendigen Q-System-Anpassungen heute an die Hand zu nehmen, um rechtzeitig gerüstet zu sein und um bei Notwendigkeit eine Q-System-Zertifizierung erfolgreich bestehen zu können. Im übrigen, je früher man beginnt, ein Q-System aufzubauen, anzupassen oder zu optimieren, desto früher erzielt das Unternehmen mehr Gewinn - und zwar Gewinn auf den verschiedensten Ebenen, wie:

- bessere Wettbewerbschancen;

- grösseres Vertrauen der Kunden;

- weniger Fehler in allen Produktentste- hungsphasen;

- geringere Fehler-, Reparatur-, Serviceund Qualitätskosten;

- höhere Produktivität;

- besseres Arbeitsklima im Unternehmen;

- höhere Motivation aller Kader, Mitarbeiterinnen und Mitarbeiter usw.

Also sicher ein erstrebenswertes und lohnenswertes Ziel für jedes einzelne Unternehmen. Dies selbstverständlich auch hinsichtlich der Realisierung des gigantischen europäischen Wirtschaftsraumes mit rund 360 Millionen Konsumenten, auch wenn die Realisierung desselben, Ende 1992 noch nicht ganz abgeschlossen sein sollte.
Chimia 46 (1992) 251-254

(c) Neue Schweizerische Chemische Gesellschaft ISSN 0009-4293

\section{Die sieben Glorreichen}

\author{
Hugo Flühler*
}

\section{Motivation}

Seit Jahren wird bei Ciba-Geigy AG sehr viel unternommen, um Arbeitsabläu$\mathrm{fe}$, Systeme und Prozesse gründlicher zu verstehen und zu beherrschen. Diese Aktivitäten werden unter dem Aspekt der Qualität und der Qualitätssicherung durchgeführt. Sie führen zwangsläufig zu besseren Prozesskenntnissen und verständlicheren Kommunikationsmethoden. All diese Aktivitäten werden schlicht mit Qualitätsmanagement bezeichnet. Gegenüber der Konkurrenz erzielen wir durch diese gepflegte Unternehmenskultur eindeutige Wettbewerbsvorteile.

Was hat der Fachbereich Statistik dem Qualitätsmanagement überhaupt anzubieten? Können die statistischen Werkzeuge in den Qualitätsbemühungen auch sinnvoll Anwendung finden? Können diese Methoden auch von allen Mitarbeitern verstanden werden?

Sofern ein Unternehmen unter Total Quality Management (TQM) nur die obligate und lästige Erfüllung von ISO-Standards und unter Statistik bloss das Nach- führen von Strichlisten versteht, sind mit statistischen Methoden kaum fruchtbare Synergismen zu erwarten.Wenn aber eine Firma oder Verwaltung TQM als Unternehmenskultur versteht und praktiziert, dann ist der Einsatz statistischer Methoden und insbesondere die Anwendung der sieben Glorreichen (Fig. 1) ein integraler Bestandteil der gelebten Geschäftsvision.

\section{Was ist TQM?}

Unter Qualität wird die Gesamtheit aller Eigenschaften und Merkmale eines Produktes oder einer Dienstleistung verstanden, um die Bedürfnisse und Erwartungen der Kunden zu erfüllen. Der Kunde jedoch lebt in einer sich stetig ändernden Umwelt und modifiziert seine Erwartungen laufend. Qualität ist als dynamischer, sich stetig ändernder Prozess zu betrachten. Was gestern noch bezüglich Qualität befriedigte, erfüllt heute schon nicht mehr die Anforderungen der Kunden.

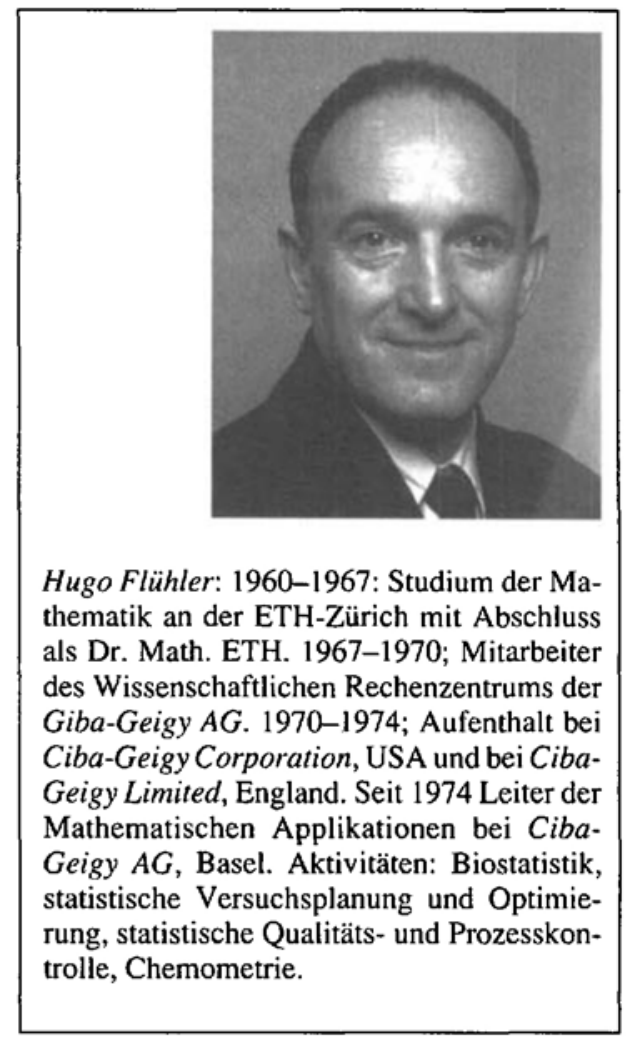

*Korrespondenz: Dr. H. Flühler Mathematische Applikationen Ciba-Geigy $A G$

R-1008.Z2.36

CH-4002 Basel 
striekonzerne mit der Umstellung auf ISO befassen. In diesen beiden Ländern entstehen ebenfalls Q-System-Zertifizierungsstellen.

Es darf somit erfreut festgestellt werden, dass auch in weiteren Teilen der Welt immer grössere Anstrengungen hinsichtlich Qualitätssicherung und der Einführung von integralen Q-Systemen sowie deren Zertifizierung unternommen werden mit dem erklärten Ziel, die Qualitätsfähigkeit der Firmen entscheidend anzuheben.

Alle diese Anstrengungen bezüglich höherer Qualitätsfähigkeit und Zuverlässigkeit, die europa- und weltweit unternommen werden, wirken sich aber teilweise sehr direkt und zum Teil auch indirekt auf die einzelnen Firmen der europäischen Industrie und Wirtschaft aus; dies schon wegen der engen europa- und weltweiten Verknüpfung der Wirtschaft.

Es gilt für die einzelnen Firmen die Herausforderung anzunehmen und die Qualitäts-und Wettbewerbsfähigkeit ganz allgemein zu steigern. Für das einzelne Unternehmen bedeutet dies, die notwendigen Q-System-Anpassungen heute an die Hand zu nehmen, um rechtzeitig gerüstet zu sein und um bei Notwendigkeit eine Q-System-Zertifizierung erfolgreich bestehen zu können. Im übrigen, je früher man beginnt, ein Q-System aufzubauen, anzupassen oder zu optimieren, desto früher erzielt das Unternehmen mehr Gewinn - und zwar Gewinn auf den verschiedensten Ebenen, wie:

- bessere Wettbewerbschancen;

- grösseres Vertrauen der Kunden;

- weniger Fehler in allen Produktentste- hungsphasen;

- geringere Fehler-, Reparatur-, Serviceund Qualitätskosten;

- höhere Produktivität;

- besseres Arbeitsklima im Unternehmen;

- höhere Motivation aller Kader, Mitarbeiterinnen und Mitarbeiter usw.

Also sicher ein erstrebenswertes und lohnenswertes Ziel für jedes einzelne Unternehmen. Dies selbstverständlich auch hinsichtlich der Realisierung des gigantischen europäischen Wirtschaftsraumes mit rund 360 Millionen Konsumenten, auch wenn die Realisierung desselben, Ende 1992 noch nicht ganz abgeschlossen sein sollte.
Chimia 46 (1992) 251-254

(c) Neue Schweizerische Chemische Gesellschaft ISSN 0009-4293

\section{Die sieben Glorreichen}

\author{
Hugo Flühler*
}

\section{Motivation}

Seit Jahren wird bei Ciba-Geigy AG sehr viel unternommen, um Arbeitsabläu$\mathrm{fe}$, Systeme und Prozesse gründlicher zu verstehen und zu beherrschen. Diese Aktivitäten werden unter dem Aspekt der Qualität und der Qualitätssicherung durchgeführt. Sie führen zwangsläufig zu besseren Prozesskenntnissen und verständlicheren Kommunikationsmethoden. All diese Aktivitäten werden schlicht mit Qualitätsmanagement bezeichnet. Gegenüber der Konkurrenz erzielen wir durch diese gepflegte Unternehmenskultur eindeutige Wettbewerbsvorteile.

Was hat der Fachbereich Statistik dem Qualitätsmanagement überhaupt anzubieten? Können die statistischen Werkzeuge in den Qualitätsbemühungen auch sinnvoll Anwendung finden? Können diese Methoden auch von allen Mitarbeitern verstanden werden?

Sofern ein Unternehmen unter Total Quality Management (TQM) nur die obligate und lästige Erfüllung von ISO-Standards und unter Statistik bloss das Nach- führen von Strichlisten versteht, sind mit statistischen Methoden kaum fruchtbare Synergismen zu erwarten.Wenn aber eine Firma oder Verwaltung TQM als Unternehmenskultur versteht und praktiziert, dann ist der Einsatz statistischer Methoden und insbesondere die Anwendung der sieben Glorreichen (Fig. 1) ein integraler Bestandteil der gelebten Geschäftsvision.

\section{Was ist TQM?}

Unter Qualität wird die Gesamtheit aller Eigenschaften und Merkmale eines Produktes oder einer Dienstleistung verstanden, um die Bedürfnisse und Erwartungen der Kunden zu erfüllen. Der Kunde jedoch lebt in einer sich stetig ändernden Umwelt und modifiziert seine Erwartungen laufend. Qualität ist als dynamischer, sich stetig ändernder Prozess zu betrachten. Was gestern noch bezüglich Qualität befriedigte, erfüllt heute schon nicht mehr die Anforderungen der Kunden.

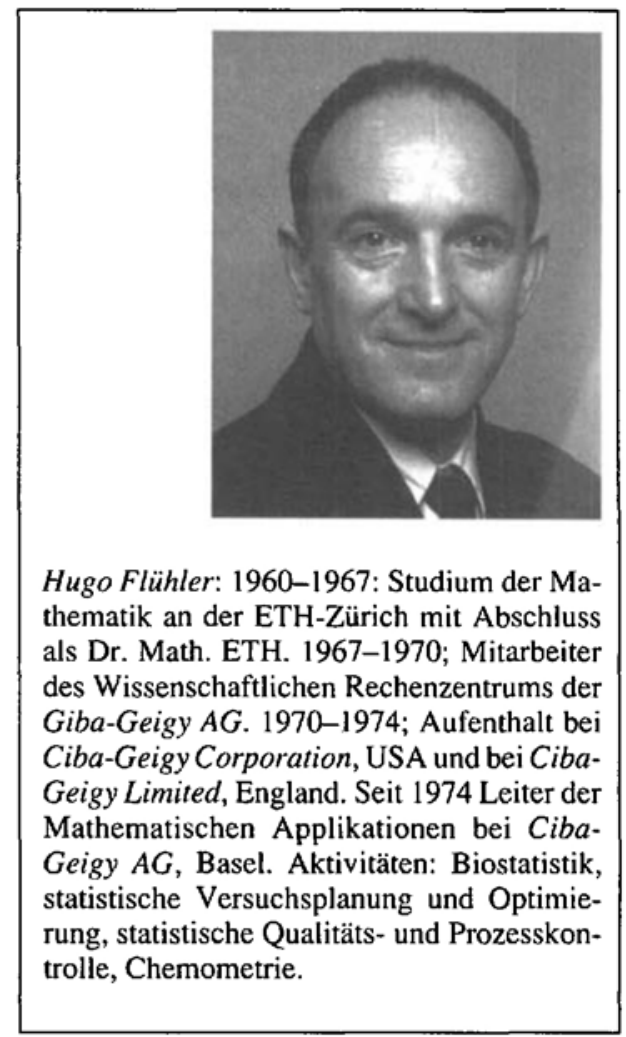

*Korrespondenz: Dr. H. Flühler Mathematische Applikationen Ciba-Geigy $A G$

R-1008.Z2.36

CH-4002 Basel 


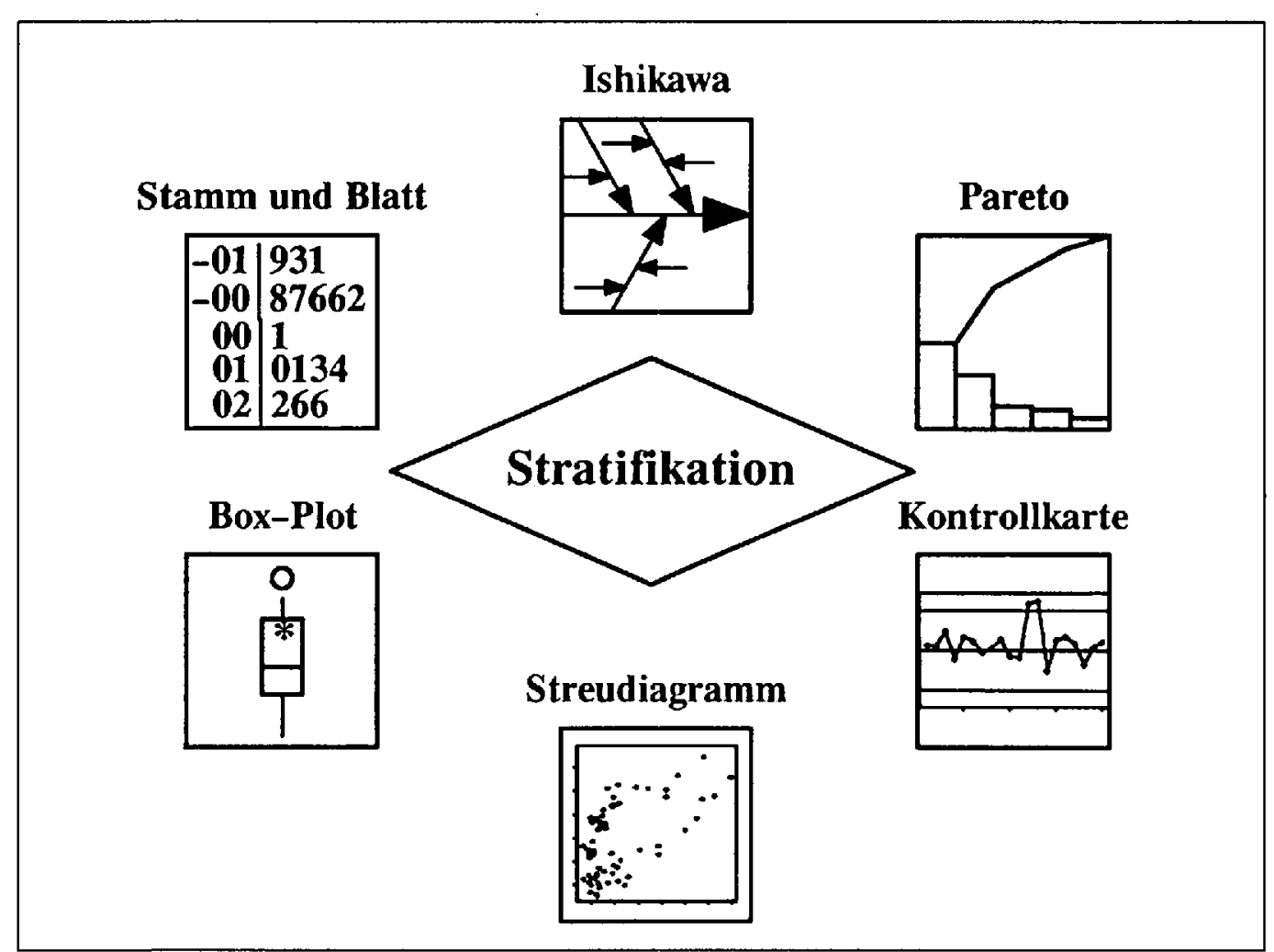

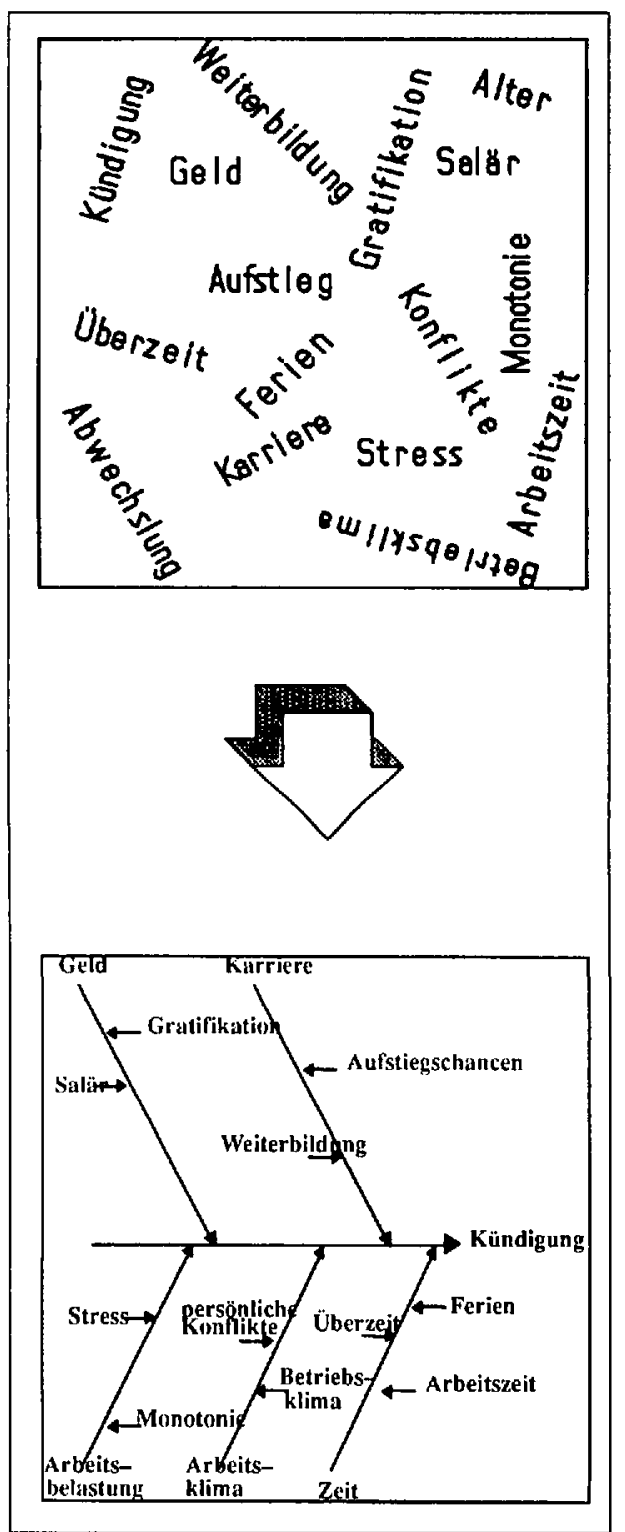

Fig. 2

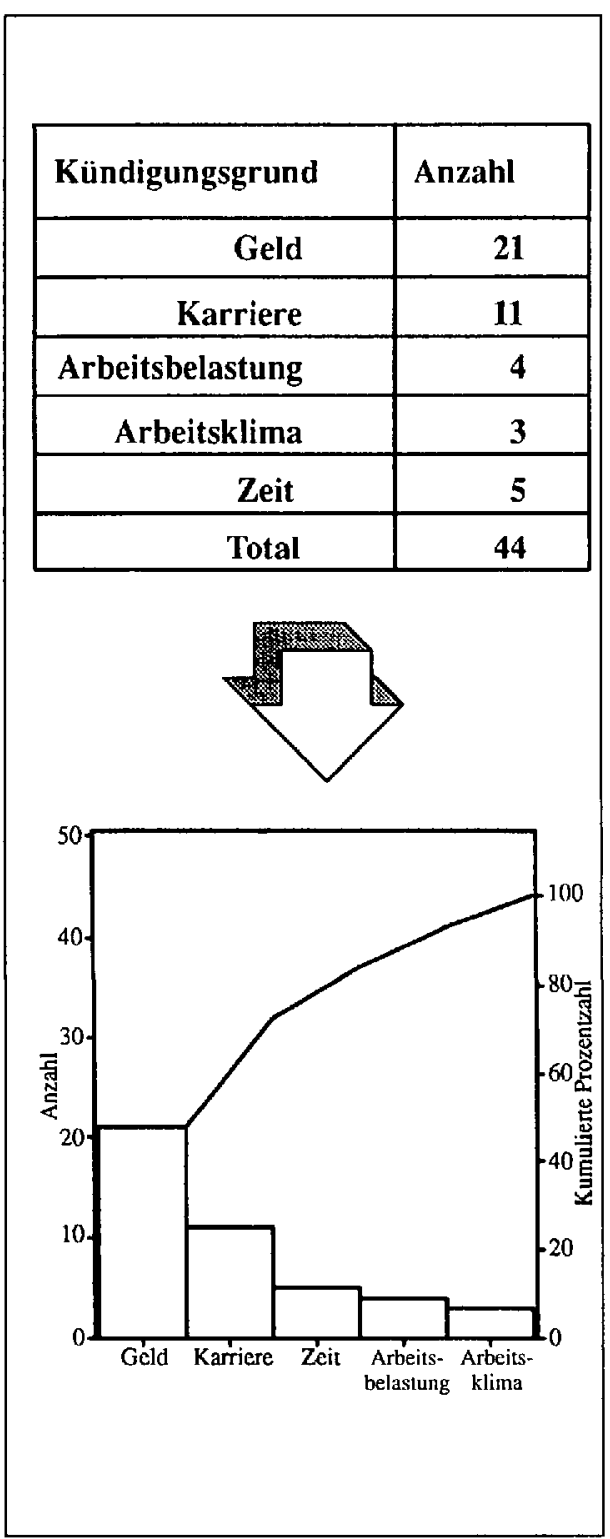

Fig. 3
Unter Quality Management wird die strategische Ausrichtung eines Unternehmens verstanden, Qualität zu erreichen, Qualitätsanforderungen zu erfüllen und fortlaufend zu verbessern. Diese visionäre Ausrichtung wird nicht nur von der Leitung gepredigt, sie wird von allen Mitarbeitern aller Stufen gelebt und gepflegt deshalb auch der Ausdruck Total Quality Management. Diese praktizierte Ausrichtung umfasst folgende Aspekte:

- Arbeitsprozesse beherrschen

(nur gründliche Kenntnisse der Arbeitsabläufe, der Zusammenhänge und Interaktionen bieten Gewähr für Qualität)

- alle organisatorischen Einheiten einbeziehen

(jeder Mitarbeiter ist involviert, ist motiviert und ist für Qualität verantwortlich)

- partnerschaftlicheZusammenarbeitmit Kunden/Lieferanten

(alle sitzen im selben Boot - der Lieferant - der Kunde - das Unternehmen und haben ein und dasselbe Ziel vor Augen, Qualität zu erzielen )

- Qualität messen und beurteilen

(Qualität kann und muss gemessen werden; Qualität garantiert auch Produktivität, da beide Grössen Hand in Hand gehen)

- Ausbildung pflegen und Statistik anwenden

(Statistische Methoden anwenden, um Ursachen zu finden und Probleme zu lösen. Gefühlsmässige Urteile sind Vorurteile.Die sieben Glorreichen sind einfache, leicht verständliche Werk- 
zeuge, die von jedem Mitarbeiter eingesetzt werden können)

\section{Die sieben Glorreichen}

Die sieben statistischen Hilfsmittel, die zur Visualisierung graphische Darstellungsformen benutzen, zeichnen sich durch ihre Einfachheit und Wirksamkeit aus. Die Werkzeuge, oftmals auch Tools genannt, unterstïtzen das Beurteilungsvermögen bei der Strukturierung von Problemen und erleichtern wesentlich die erforderliche Kommunikation. Da Qualität ja gemessen werden muss und dadurch grosse Datenmengen erzeugt werden, helfen diese Tools speziell in den Datenhaufen zur Information zu finden. Wir unterscheiden vier unterschiedliche Aktivitätenmuster, je nach Problemstellung, und stellen sie im folgenden kurz vor. Es sind dies

- Begriffe und Fakten sammeln und ordnen

- Zahlen inspizieren und beurteilen

- Abläufe beobachten und kontollieren

- Abhängigkeiten untersuchen und sichtbar machen

\section{Fakten ordnen}

Den Schlüssel zum Erfolg stellen in einem Unternehmen die Mitarbeiter dar. Aus diesem Grunde werden mit Hilfe von Austrittsinterviews nach den Ursachen der Kündigungen befragt. Die verschiedenen Erklärungen sind in Fig. 2 unstrukturiert (oben) und strukturiert (unten) dargestellt. Die untere Darstellung, welche die Fakten geordnet nach Unter- und Oberbegriffen anordnet und visuell darstellt, ist das Ishikawa-Diagramm.

Das Diagramm dient zur Strukturierung eines Problems und unterstützt den zielbezogenen Denk- und Kommunikationsprozess. Ein Vergleich der beiden Darstellungen zeigt offensichtlich, dass der Kommunikationsprozess mit dem Ishika$w a$-Diagramm bedeutend besser unterstützt wird. Verständlichere Kommunikationsprozesse fördern Problemverständnis und Qualität.

Häufig werden Ergebnisse tabellarisch dargestellt, wie das in Fig. 3 (oben) für das Austrittsinterview gezeigt ist. Bedeutend informativer hingegen stellt das ParetoDiagramm (unten) denselben Sachverhalt dar und vermittelt die Prioritäten für die Problemanalyse. Das Pareto-Diagramm basiert auf der Erkenntnis, dass oft wenige Faktoren zahlenmässig viel zu einem Problem beitragen. Diese Information wird in Form eines Balkendiagramms, der Grösse nach geordnet, dargestellt.

Diese beiden vorgestellten Tools sind einfache und leicht verständliche Werkzeuge, um Situationen, Probleme oder

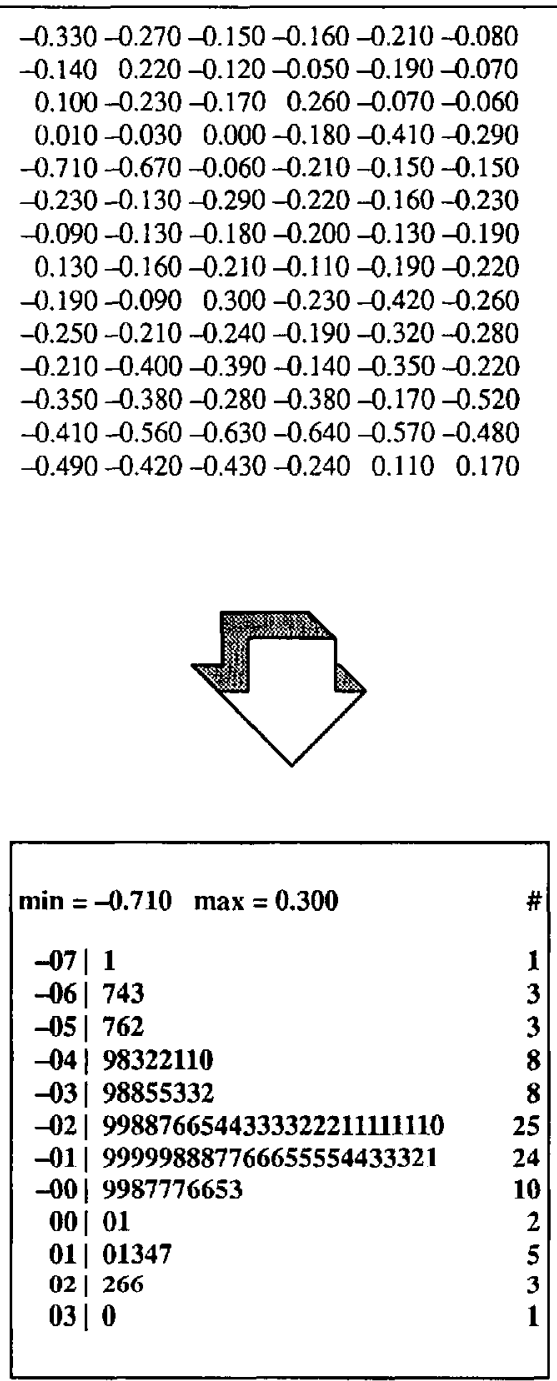

Fig. 4

Ereignisse zu ordnen, zu strukturieren und in leicht verständlicher, visualisierter Form zu kommunizieren.

\section{Zahlen inspizieren}

Viele Zahlen ergeben lediglich einen Datenhaufen, der an und für sich in tabellarischer Form noch keine sinnvolle Information darstellt (Fig. 4, oben). Die Stammund Blatt-Darstellung (Fig. 4, unten) ist hingegen ein geeignetes Hilfsmittel, um Datenhaufen visuell derart zu vermitteln, dass die Daten für den Betrachter auch Informationen beinhalten. Diese Darstellung kann einfach mit Papier und Bleistift realisiert werden und erweckt - obwohl als Tabelle dargestellt-einen graphischen Eindruck.

Die Darstellung vermittelt wertvolle Erkenntnisse bezüglich Verteilung, orientiert über mögliche Ausreisser oder verschiedene Populationen.

Grosse Datenhaufen (Fig.5, oben) können auch informativ durch den Box-Plot, das heisst durch fünf Kennzahlen charakterisiert werden (Fig.5, unten). Diese fünf
$-0.330-0.270-0.150-0.160-0.210-0.080$

$-0.140-0.220-0.120-0.050-0.190-0.070$

$0.100-0.230-0.170 \quad 0.260-0.070-0.060$ $0.010-0.030 \quad 0.000-0.180-0.410-0.290$ $-0.710-0.670-0.060-0.210-0.150-0.150$ $-0.230-0.130-0.290-0.220-0.160-0.230$ $-0.090-0.130-0.180-0.200-0.130-0.190$ $0.130-0.160-0.210-0.110-0.190-0.220$ $-0.190-0.090 \quad 0.300-0.230-0.420-0.260$ $-0.250-0.210-0.240-0.190-0.320-0.280$ $-0.210-0.400-0.390-0.140-0.350-0.220$ $-0.350-0.380-0.280-0.380-0.170-0.520$ $-0.410-0.560-0.630-0.640-0.570-0.480$

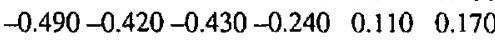
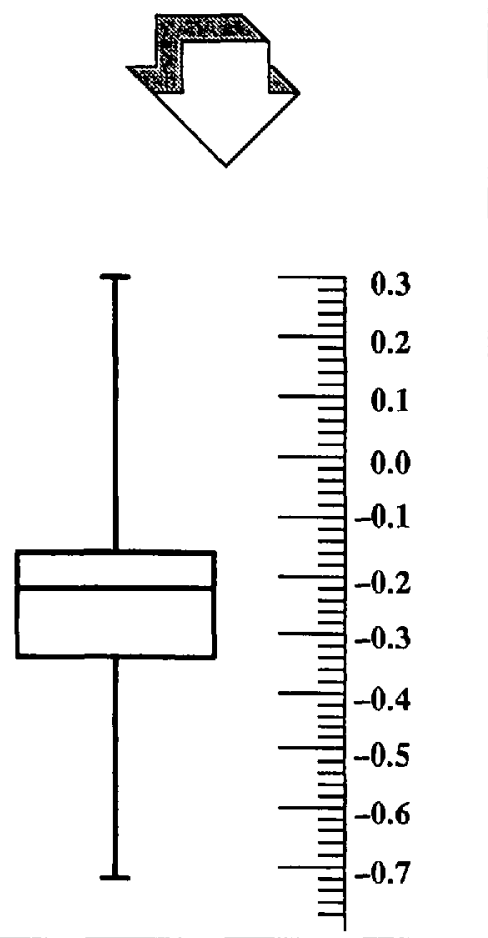

Fig. 5

Kennzahlen sind der grösste und der kleinste Wert sowie drei weitere Werte, die den geordneten Datenhaufen in vier gleichgrosse Haufen unterteilen. Die Kennzahlen werden graphisch in Form eines Kastens mit Fühlern dargestellt und dienen vor allem zur Verdichtung von grossen Datenhaufen.

Diese beiden statistischen Werkzeuge dienen hauptsächlich dazu, die brachliegenden Datenfriedhöfe in wertvolle Wissensbasen umzuwandeln. Dass man sich bei diesem Verdichtungsprozess wiederum der visuellen Fähigkeit des menschlichen Auges bedient, ist mit ein Grund für den grossen Erfolg, den diese beiden Methoden in ihrer Anwendung verbuchen.

\section{Abläufe kontrollieren}

Um zeitliche Abläufe zu beobachten und zu kontrollieren, taugen tabellarische Darstellungen, wie das in Fig. 6 (oben) vermittelt wird, nicht. Erst eine zeitabhängige Graphik in Form einer sogenannten Kontrollkarte vermag den Prozess visuell derart darzustellen, dass relevante Infor- 
mation sichtbar wird (Fig. 6, unten). Entsprechende Referenzwerte als obere und untere Grenzen ermöglichen die Identifikation von Ausreissern oder eine Antwort auf die Frage: Ist der Prozess unter Kontrolle? Die Kontrollkarte vermittelt einen zeitabhängigen Prozess im Rahmen der zu erwartenden Schwankungen.

\section{Grössen verknüpfen}

Die sogenannte Korrelation ist ein mathematisches Mass für die lineare Beziehung zweier Grössen. Eine tabellarische Darstellung zweier Grössen wie z.B. das Gewicht und der Preis von Personenwagen (Fig. 7, oben) vermag nicht über den Zusammenhang zu informieren. Auch

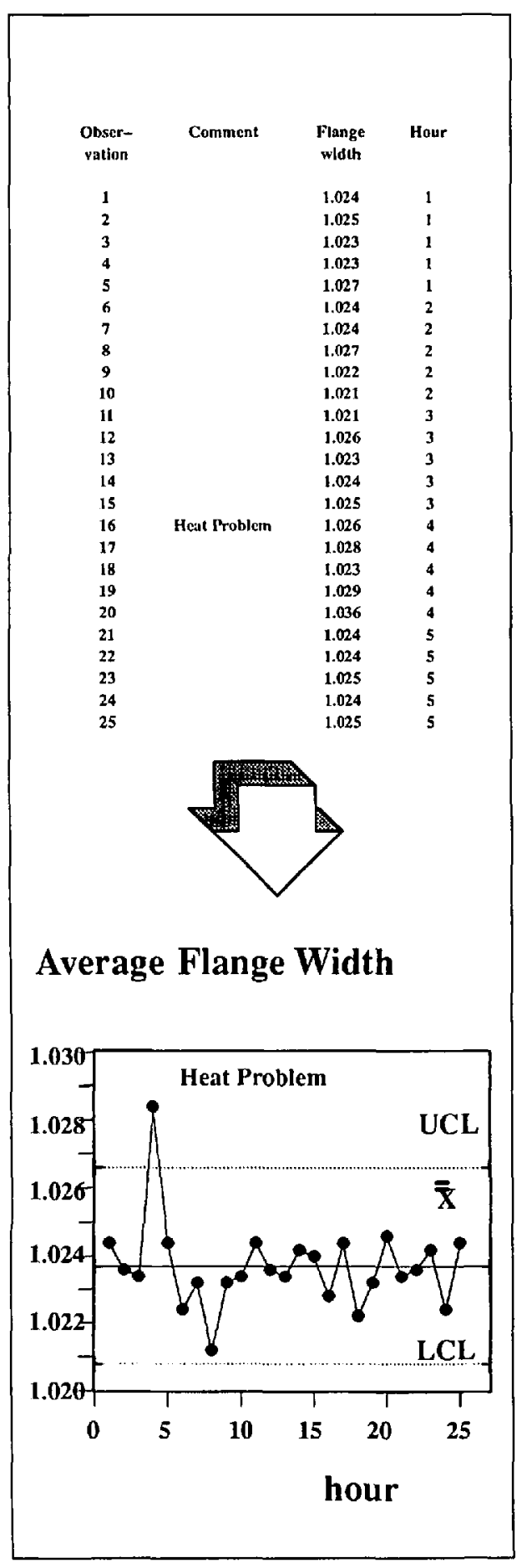

Fig. 6 eine isolierte Beurteilung der berechneten Korrelation kann häufig zu völlig falschen Interpretationen führen. Bedeutend nützlichere Eindrücke liefert die visuelle Darstellung der Beziehung zweier Grössen mit Hilfe des Streudiagramms (Fig. 7, unten). Diese Darstellung liefert die visuelle Grundlage, sich über die Art und Form des Zusammenhangs ein Bild zu verschaffen.

Als sogenannte Meta-Methode kann die Stratifikation betrachtet werden, die mehr eine statistische Strategie darstellt und jeweils in Kombination mit den bereits vorgestellten Hilfsmitteln Anwendung findet. Durch die Stratifikation werden vorhandene Daten oder Fakten nach

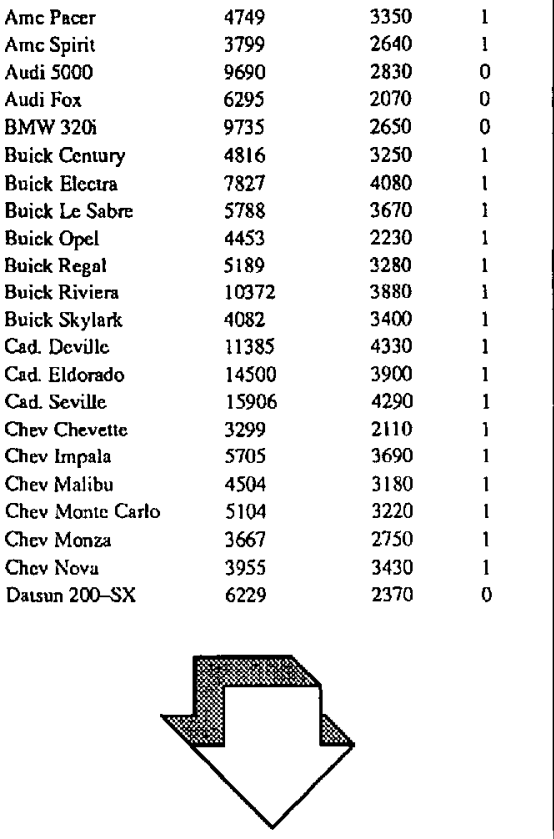

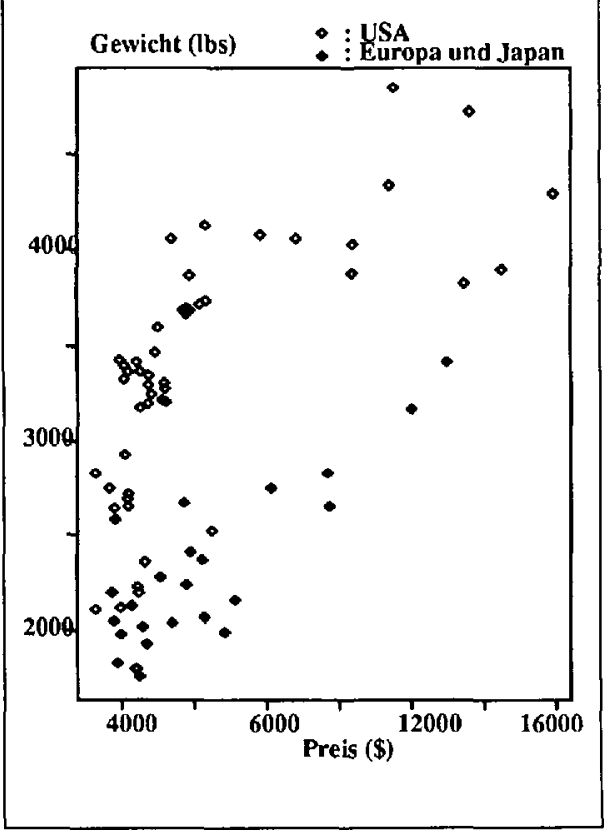

Fig. 7 unterschiedlichen Gesichtspunkten betrachtet. Das Steudiagramm in Fig. 7, stratifiziert nach amerikanischen und nichtamerikanischen Autos, vermittelt für die beiden Populationen unterschiedliche $\mathrm{Zu}$ sammenhänge. Ohne Stratifikation würde das Streudiagramm kaum relevanten $\mathrm{Zu}$ sammenhang $\mathrm{z}$ wischen Gewicht und Preis offenbaren.

\section{Strategischer Ausblick}

Ein Blick nach Japan, zu unserem grossen Lehrmeister für Qualität und Produktivität, vermittelt uns den wirklichen Sachverhalt.

\section{Japan tut es, wir noch nicht!}

Gemeint ist die praktische Anwendung statistischer Prinzipien in der japanischen Industrie.

Alle Mitarbeiter eines Unternehmens in Japan verstehen die sieben statistischen Werkzeuge. Die Japaner beherrschen und setzen diese Tools ein, sie bedienen sich dieser einfachen Werkzeuge zur förderung der Kommunikation und beim Problemlösungsprozess. Die japanische Industrie betreibt ein intensives Ausbildungsprogramm in Industrieller Statistik. Interne Ausbildungskurse von bis zu 30 Tagen werden in Qualitäts- und Prozesskontrolle, in statistischer Versuchsplanung und Optimierung sowie im 'Quality by Design' durchgeführt (Science (Editorial) 1989, 244).

Wo stehen wir in der Schweiz? Noch weit entfernt vom statistischem Ausbildungsstand der japanischen Industrie. Statistik ist bei uns kaum bekannt oder wird nur von Spezialisten angewandt. Praxisbezogene Ausbildung wird kaum angeboten.

Diese Situation hat uns, die Mathematischen Applikationen, bewogen, sogenannte Technologietransfer-Kurse anzubieten, und zwar Ciba-Geigy intern wie auch auf dem freien Markt. Es sind dies:

Die sieben Glorreichen

als 1-Tageskurs für alle Mitarbeiter eines Unternehmens zur Förderung des Qualitätsgedankens und zur Sicherung des Arbeitsablaufs, und

Industrielle Statistik in der Qualitätstechnologie

als 4-Tageskurs für Verfahrens-Chemiker und Ingenieure. 